\title{
Abbreviated MRI for HCC surveillance: is it ready for clinical use?
}

\author{
Naik Vietti Violi ${ }^{1} \cdot$ Bachir Taouli $^{2,3}$ (D) \\ Received: 20 March 2020 / Accepted: 21 April 2020 / Published online: 12 May 2020 \\ (C) European Society of Radiology 2020
}

\section{Key Points}

- Abbreviated MRI (AMRI) protocols consist of acquiring only a minimal number of MRI sequences for HCC surveillance with acceptable diagnostic performance compared to complete MRI.

- AMRI protocol options include non-contrast AMRI, dynamic AMRI or hepatobiliary phase AMRI post gadoxetate injection.

- The best AMRI protocol for HCC surveillance needs to be defined in a large multicentre prospective study.

Hepatocellular carcinoma (HCC) is the second leading cause of cancer-related death worldwide. Clinical practice guidelines recommend HCC screening and surveillance in patients at risk (patients with cirrhosis and chronic HBV) for early detection and treatment, despite the limited number of highquality studies demonstrating the effectiveness of HCC screening in decreasing mortality [1]. Specifically, the EASL (European Association for the Study of the Liver) guidelines recommend ultrasound (US) every 6 months [2], while the AASLD (American Association for the Study of Liver Diseases) recommend US with or without serum alpha fetoprotein (AFP) every 6 months in patients at risk [3].

However, US has shown limited ability for detection of small HCC, around $47 \%$, especially in patients with large body habitus and/or advanced cirrhosis [4]. A recent Korean prospective study reported even lower sensitivity of US (27.9\%) for HCC detection in the context of screening [5]. To improve the performance of imaging screening/surveillance, many centres perform CT or MRI exams, which are not necessarily optimal for HCC surveillance due to radiation

This comment refers to the article available at https://oi.org/10.1007/ s00330-020-06754-4.

Bachir Taouli

bachir.taouli@mountsinai.org

1 Department of Radiology, Lausanne University Hospital, Lausanne, Switzerland

2 Department of Diagnostic, Molecular and Interventional Radiology, Icahn School of Medicine at Mount Sinai, New York, NY, USA

3 BioMedical Engineering and Imaging Institute, Icahn School of Medicine at Mount Sinai, 1470 Madison Avenue, New York, NY 10029, USA exposure (for CT) and higher cost and long exam duration for MRI (between 20 and $40 \mathrm{~min}$ ).

This has recently fuelled interest in abbreviated MRI (AMRI) protocols which consist of acquiring only a few select MRI sequences to aim for a reduction in time while maintaining acceptable diagnostic performance for HCC detection. Several protocols/combinations of MRI sequences have been proposed, including a non-contrast (NC)-AMRI protocol combining T2-weighted imaging (T2wi) and diffusionweighted imaging (DWI) [6, 7], dynamic (Dyn)-MRI with an extracellular gadolinium-based contrast agent [8] or gadoxetate-enhanced T1wi obtained during the hepatobiliary phase (HBP) with T2wi with/without DWI [6, 9]. The idea is to recall patients for $\mathrm{CT}$ or complete contrast-enhanced MRI in positive NC-AMRI and HBP-AMRI studies.

The current study by Whang et al [10] compared the diagnostic performance of NC-AMRI and HBP-AMRI for HCC detection in a population of patients with and without HCC. The authors included 140 patients with early HCC (according to Milan criteria) with gadoxetate-enhanced MRI and 123 patients without HCC used as controls. Two independent readers reviewed the 2 datasets (NC-AMRI and HBPAMRI) extracted from the complete MRI. There was equivalent per-patient sensitivity $(85.7 \%$ and $86.4 \%$ for NC-AMRI, and $90 \%$ and $89.3 \%$ for HBP-AMRI, for readers 1 and 2, respectively) and specificity ( $92.7 \%$ for both datasets and readers). The authors concluded that NC-AMRI and HBPAMRI are equivalent for detection of early HCC.

This study unveiled important information about HCC detection with AMRI. The authors focused on the comparison between two different AMRI protocols, demonstrating the diagnostic value of AMRI for HCC screening, and its likely higher diagnostic performance compared to US, with the need to define the best AMRI protocol. In previous studies, NC- 
AMRI showed excellent sensitivity (79.1-85.5\%) and specificity $(94.9-97.9 \%)$ [6, 7]. Reconstructed HBP-AMRI with gadoxetate Whang reported HCC detection sensitivity of $79.6-85.7 \%$ and specificity of $91.2-97.3 \%$, in diagnostic cohorts rather than screening cohorts, with no difference in sensitivity for HCC detection between diagnostic MRI and AMRI $[6,9]$. The performance of Dyn-AMRI for HCC detection has been reported recently in a nonscreening population (with average per-patient sensitivity of $92.1 \%$ and specificity of $88.7 \%$ ) [8]. When considering comparison with US, Park et al. recently published results on a prospective comparison of NC-AMRI to US in patients at high risk for HCC [7]. The authors demonstrated sensitivities of $27.9 \%$ for US and $79.1 \%$ for NC-AMRI, with a significant difference between US and NC-AMRI. Tillman et al. compared HBP-AMRI to US and concluded that HBP-AMRI provides higher sensitivity (85.2\%) compared to reported US values [11].

Of note, the present study has two shortcomings that should be mentioned. First, the population assessed was not a screening population (more than $50 \%$ of patients had HCC), while HCC prevalence ranges typically between 0.25 and $7.6 \%$ in screening populations. Of note, this limitation is present in most published AMRI studies. This may overestimate the performance of AMRI. Second, the study included mainly HBV patients, which is not necessarily representative of the Western population at risk of HCC. This may limit generalization of study results.

It is currently difficult to define the best AMRI protocol for HCC screening and surveillance. In addition to diagnostic performance, one needs to consider feasibility, adoption, and cost/cost-effectiveness. From a practical point of view, NCAMRI presents clear advantages over contrast-enhanced AMRI, by avoiding the need for contrast injection, which simplifies and shortens even more the protocol, and removes the potential risks of gadolinium-based contrast agents (GBCAs), namely nephrogenic systemic fibrosis and gadolinium tissue deposition. Contrast-enhanced AMRI including HBP-AMRI and Dyn-AMRI requires intra-venous GBCA injection, and seems to provide higher sensitivity even if statistical difference is not demonstrated, such as in the present study. With HBP-AMRI, the injection can be performed before the exam outside the AMRI room with acquisition at 20 min after contrast injection, which simplifies the injection process. HBP-AMRI is a highly sensitive sequence for HCC detection, however with the risk of false positives. DynAMRI, on the other hand, has the advantage of providing detection and characterization during the same exam which is not possible with NC-AMRI and HBP-AMRI where a recall CT or complete MRI is required in positive cases. Additionally, the cost of extracellular GBCA is lower than that of gadoxetate. A recent cost-effectiveness study comparing HCC surveillance with US, CT, complete MRI, and AMRI concluded that HCC screening performed with AMRI was cost-effective in a conservative scenario ( $52 \%$ surveillance compliance) [12].

While the most accurate and feasible AMRI protocol and the target population for AMRI still need to be clarified, AMRI is emerging as a promising tool to be used as an alternate to US for HCC surveillance. However, widespread use of AMRI will still be limited by MRI access/availability and potential contra-indications, compared to US.

Future large multicentre prospective studies in HCC screening population rather than diagnostic population comparing US with different AMRI protocols are needed to determine the AMRI diagnostic performance. The added value of circulating tumour biomarkers such as circulating tumour DNA should be assessed. Advanced cost-effectiveness analysis with models including each region population characteristics (HCC prevalence, type of chronic liver disease, body habitus) and radiologic exam costs will also be needed in order to implement AMRI in clinical practice.

Funding information Naik Vietti Violi received financial support from the Swiss National Science Foundation, fellowship P2LAP3_178053. Bachir Taouli received financial support from Bayer.

\section{Compliance with ethical standards}

Guarantor The scientific guarantor of this publication is Bachir Taouli.

Conflict of interest The authors of this manuscript declare relationships with the following companies:

Bachir Taouli:

Research grants: Bayer Healthcare, Takeda

Consultant: Bayer Healthcare, Alexion

The other authors of this manuscript declare no relationships with any companies whose products or services may be related to the subject matter of the article.

Statistics and biometry Not applicable

Informed consent Not applicable

Ethical approval Not applicable

Methodology Not applicable

\section{References}

1. Zhang BH, Yang BH, Tang ZY (2004) Randomized controlled trial of screening for hepatocellular carcinoma. J Cancer Res Clin Oncol $130: 417-422$

2. European Association for the Study of the Liver (2018) EASL Clinical Practice Guidelines: Management of hepatocellular carcinoma. J Hepatol 69:182-236

3. Marrero JA, Kulik LM, Sirlin CB et al (2018) Diagnosis, staging, and Management of Hepatocellular Carcinoma: 2018 Practice Guidance by the American Association for the Study of Liver Diseases. Hepatology 68:723-750 
4. Tzartzeva K, Obi J, Rich NE et al (2018) Surveillance imaging and alpha fetoprotein for early detection of hepatocellular carcinoma in patients with cirrhosis: a meta-analysis. Gastroenterology 154 : 1706-1718 e1701

5. Kim SY, An J, Lim YS et al (2017) MRI with liver-specific contrast for surveillance of patients with cirrhosis at high risk of hepatocellular carcinoma. JAMA Oncol 3:456-463

6. Besa C, Lewis S, Pandharipande PV et al (2017) Hepatocellular carcinoma detection: diagnostic performance of a simulated abbreviated MRI protocol combining diffusion-weighted and T1weighted imaging at the delayed phase post gadoxetic acid. Abdom Radiol (NY) 42:179-190

7. Park HJ, Jang HY, Kim SY et al (2020) Non-enhanced magnetic resonance imaging as a surveillance tool for hepatocellular carcinoma: comparison with ultrasound. J Hepatol 72:718-724

8. Khatri G, Pedrosa I, Ananthakrishnan L et al (2020) Abbreviatedprotocol screening MRI vs. complete-protocol diagnostic MRI for detection of hepatocellular carcinoma in patients with cirrhosis: an equivalence study using LI-RADS v2018. J Magn Reson Imaging $51: 415-425$
9. Marks RM, Ryan A, Heba ER et al (2015) Diagnostic per-patient accuracy of an abbreviated hepatobiliary phase gadoxetic acidenhanced MRI for hepatocellular carcinoma surveillance. AJR Am J Roentgenol 204:527-535

10. Whang S, Choi MH, Choi JI, Youn SY, Kim DH, Rha SE (2020) Comparison of diagnostic performance of non-contrast MRI and abbreviated MRI using gadoxetic acid in initially diagnosed hepatocellular carcinoma patients: a simulation study of surveillance for hepatocellular carcinomas. Eur Radiol. https://doi.org/10.1007/ s00330-020-06754-4

11. Tillman BG, Gorman JD, Hru JM et al (2018) Diagnostic per-lesion performance of a simulated gadoxetate disodium-enhanced abbreviated MRI protocol for hepatocellular carcinoma screening. Clin Radiol 73:485-493

12. Lima PH, Fan B, Berube J et al (2019) Cost-utility analysis of imaging for surveillance and diagnosis of hepatocellular carcinoma. AJR Am J Roentgenol:1-9

Publisher's note Springer Nature remains neutral with regard to jurisdictional claims in published maps and institutional affiliations. 